\title{
EVOLUTION OF SIZE AND MORPHOLOGY OF $\gamma$ ' PRECIPITATES IN UDIMET 720 LI DURING CONTINUOUS COOLING
}

\author{
R. Radis ${ }^{1}$, M. Schaffer ${ }^{2}$, M. Albu ${ }^{2}$, G. Kothleitner ${ }^{2}$, P. Pölt ${ }^{2}$ und E. Kozeschnik ${ }^{1,3,4}$ \\ ${ }^{1}$ Christian Doppler Laboratory for Early Stages of Precipitation, Graz University of Technology, \\ Kopernikusgasse 24, A-8010 Graz, Austria \\ ${ }^{2}$ Research Institute for Electron Microscopy, Graz University of Technology, \\ Steyrergasse 17, A-8010 Graz, Austria \\ ${ }^{3}$ Institute for Materials Science and Welding, Graz University of Technology, \\ Kopernikusgasse 24, A-8010 Graz, Austria \\ ${ }^{4}$ Materials Center Leoben Forschung GmbH,Roseggerstraße 12, A-8700 Leoben, Austria
}

Keywords: multimodal, nickel based alloy, gamma prime, precipitation kinetics

\begin{abstract}
In this work, an analysis of the evolution of precipitates in the nickel-base superalloy UDIMET $720 \mathrm{Li}$ during continuous cooling is carried out. Starting from the solution-annealed condition, the precipitation kinetics, the distribution and the morphology of particles during continuous cooling is studied experimentally by SEM, EFTEM and FIB. The results are compared to simulations using the software MatCalc. Depending on the cooling rate, discontinuous precipitation as well as monomodal and multi-modal distributions are observed. The precipitate microstructure of various samples is investigated and evaluated quantitatively to determine the precipitate distribution parameters. The experimental results are discussed and compared with the results of the simulation. Finally, two parameter studies are carried out which indicate the sensitivity of the simulation results on the input data, i.e. the thermodynamic and diffusion databases.
\end{abstract}

\section{Introduction}

The term superalloy is generally used for a large amount of different alloys. The major motivation for development of these materials is the fabrication of gas turbines for aircrafts and power plants. For a high degree of efficiency, these machines require combustion at elevated temperatures. Usually, these alloys combine high strength and good corrosion resistance, which are essential for application in modern gas turbine aero-engines, e.g. for high-speed rotating components, such as high pressure turbine disks.

A good control of the technological properties of these alloys is inherently related to a proper control of the precipitation of a large amount of fine-dispersed coherent $\mathrm{Ni}_{3}(\mathrm{Al}, \mathrm{Ti})$ particles $\left(\gamma^{\prime}\right.$ precipitates) with ordered $\mathrm{Ll}_{2}$ structure in a face-centred cubic $\gamma$ matrix. The main strengthening mechanism in these materials is precipitation hardening. Depending on the cooling rate, the shape of these particles differ in a wide range, from simple spheres over cubes, octocubes to complex structures such as octodendrites and dendrites. This large variety of dissimilar morphologies, founded in dissolution and precipitation processes, is well known and documented in several articles, e.g. [1-11].
It is also well known that bimodal distributions of $\gamma^{\prime}$ precipitates in a $\gamma$ matrix can lead to superior mechanical properties in these alloys [12-14]. Primary $\gamma^{\prime}$ precipitates arranged on grain boundaries prevent the growth of $\gamma$-grains during solution heat treatment. A second population of these precipitates appear intergranularly and is responsible for a high strength. Such microstructures are usually achieved by special multi-stage heat treatments, which are generally complex and costly. Some authors have already shown that such bimodal, or even multimodal, size distributions of $\gamma^{\prime}$ particles can also be achieved by a series of $\gamma^{\prime}$ nucleation bursts during continuous cooling from the solution annealed state [15-22]. Thereby, the individual particle populations precipitate at different temperatures and from different chemical compositions of the $\gamma$ matrix.

Studying these nucleation and diffusion processes experimentally is expensive, especially in nickel based superalloys. With the development of specialized simulation software, e.g. the software MatCalc [23-25], it is possible, nowadays, to investigate these complex processes with computational methods on the researchers desktop.

J. Mao et.al. [20] carried out investigations of the evolution of multimodal $\gamma^{\prime}$ precipitate size distributions during continuous cooling in UDIMET $720 \mathrm{Li}$. However, his experimental methods have concentrated on SEM investigations, thus not capturing very fine $\gamma^{\prime}$ precipitates. These authors suggested to do further investigations with high resolution TEM microscopes.

In the present work both, experimental investigations using highresolution techniques as well as theoretical simulations using the software MatCalc are applied to investigate the evolution of $\gamma$ ' precipitates in the Ni-base superalloy UDIMET $720 \mathrm{Li}$ during continuous cooling. A series of experiments with varying cooling rates is performed and the results of the experimental investigation are compared to the predictions of the simulation. Furthermore, the theoretical background for the formation of multimodal size distributions of precipitates is presented and confirmed by experimental results where we identified monomodal, bi-modal and tri-modal $\gamma^{\prime}$ size distributions.

Table I: Chemical Composition of UDIMET $720 \mathrm{Li}$ in wt\% [26]

\begin{tabular}{ccccccccccc}
\hline $\mathrm{Cr}$ & $\mathrm{Ni}$ & $\mathrm{Co}$ & $\mathrm{Mo}$ & $\mathrm{Ti}$ & $\mathrm{Al}$ & $\mathrm{W}$ & $\mathrm{Fe}$ & $\mathrm{C}$ & $\mathrm{Mn}$ & $\mathrm{B}$ \\
\hline 15.92 & 57.6 & 14.43 & 2.96 & 4.96 & 2.53 & 1.26 & 0.09 & 0.014 & 0.02 & 0.014 \\
\hline
\end{tabular}


The chemical composition of UDIMET $720 \mathrm{Li}$ was analysed using emission spectroscopy. The results are given in Table I in wt $\%$.

\section{Experiments and Simulation}

\section{Experimental}

The precipitate microstructure of 13 samples is evaluated qualitatively and quantitatively to determine the precipitate distribution parameters and the morphology of the precipitates. The samples are cut into cylindrical pieces of $ø 10 \times 10 \mathrm{~mm}$, except for two samples of size $ø 5 \times 10 \mathrm{~mm}$ to achieve the fastest cooling rates. Each sample is soaked at $1180{ }^{\circ} \mathrm{C}$ for $6 \mathrm{~h}$ and then quenched into water. In references $[19,20]$, the super-solvus solution temperature is recommended with approximately $\mathrm{T}_{\mathrm{sol}}=1160{ }^{\circ} \mathrm{C}$. Scanning electron microscopic analyses have shown that this heat treatment is suitably for achieving solid solution condition and dissolving all precipitates.

Depending on the cooling rate, which is varied between $78 \mathrm{~K} / \mathrm{s}$ and $0,00416 \mathrm{~K} / \mathrm{s}$, different equipment is used for different heat treatments. The samples are heated to a temperature of $1180{ }^{\circ} \mathrm{C}$ under argon atmosphere as fast as possible, kept on this level for 30 minutes to dissolve precipitates which formed temporarily before reheating, and then cooled continuously to $400{ }^{\circ} \mathrm{C}$ at different cooling rates (see Table II). Finally, they are quenched into water to suppress further precipitation. Afterwards, the distribution and the morphology of precipitates are studied by SEM, EFTEM and FIB. The relevant precipitation parameters are determined using the stereological correction procedure after Sonderegger [27]. In each case, more than 100 precipitates are measured to determine the distributions. For this correction, SEM penetration depth data were used from reference [28].

Table II: Continuous cooling rates of the present investigation

\begin{tabular}{|c|l|r|}
\hline sample & $\begin{array}{c}\text { cooling rate } \\
{\left[{ }^{\circ} \mathrm{C} / \mathrm{s}\right]}\end{array}$ & $\begin{array}{c}\text { cooling time } \\
{[\mathrm{s}]}\end{array}$ \\
\hline 1 & 78 & 10 \\
2 & 19.5 & 40 \\
3 & 6.5 & 120 \\
4 & 3.25 & 240 \\
5 & 1.625 & 480 \\
6 & 0.867 & 900 \\
7 & 0.433 & 1800 \\
8 & 0.217 & 3600 \\
9 & 0.03333 & 23400 \\
10 & 0.01667 & 46800 \\
11 & 0.00984 & 79200 \\
12 & 0.00619 & 126000 \\
13 & 0.00416 & 187200 \\
\hline
\end{tabular}

\section{Electron microscopy}

The backscattered electron (BSE) images are recorded from polished cross sections by use of a 4-quadrant backscattered electron detector attached to a Zeiss DSM 982 Gemini scanning electron microscope (SEM). With decreasing energy of the primary electrons also the interaction volume of the electrons with the material decreases, thus enabling the detection of precipitates of decreasing size. But with decreasing electron energy also the detection sensitivity of the BSE detector decreases. Thus, as a compromise electron energies between 7 and $10 \mathrm{keV}$ are used for the recording of the BSE images.
Energy filtered transmission electron microscopy (EFTEM) investigations are carried out on a Philips CM20/Scaning TEM (STEM) and a FEI Tecnai F20 both equipped with Gatan imaging filters (GIF). The microscopes are operated at $200 \mathrm{kV}$ with a $\mathrm{LaB}_{6}$ cathode and a Shottki field emitter, respectively. The pictures are recorded with the slow scan CCD camera integrated in the GIF with a local resolution of about $1 \mathrm{~nm}$. All images are processed with Gatan's Digital Micrograph, and corrected for drift, dark current and gain variations. Electron energy loss spectroscopy (EELS) and X-ray dispersive spectroscopy (EDX) are used for the precipitate analysis.

A dual beam-focused ion beam (DB-FIB) consisting of both, an ion and an electron source, offers a unique way for $3 \mathrm{D}$ analysis by sequentially milling the sample, and analyzing each freshly produced surface. From the series of data created in this way, a $3 \mathrm{D}$ volume model can be reconstructed. The fully automated 3DEDXS method [36] is performed using the Nanolab Nova200 DBFIB (FEI) and the Genesis EDXS detector (EDAX). The DB-FIB is operated with an ion beam current of $1 \mathrm{nA}$ at $30 \mathrm{kV}$ acceleration voltage during milling, and with an electron beam current of $2.2 \mathrm{nA}$ at $15 \mathrm{kV}$ acceleration voltage during SEM and EDXS analysis. The data is evaluated using customized routines such as image registration and adaptive threshold image segmentation before visualizing the models with the Amira software (Mercury Computer Systems SA). In the course of the experiment, a total sample volume of 4,45 x $6,18 \times 2,55 \mu^{3}$ is reconstructed with a voxel size of $35 \times 45 \times 150 \mathrm{~nm}^{3}$.

\section{Computer simulations}

For the theoretical analysis of the precipitation behaviour in UDIMET $720 \mathrm{Li}$, the simulation software MatCalc [23-25] is used. This programme uses a novel theoretical approach that has been developed for simulation of precipitation kinetics in general, multi-component alloys. The theoretical foundation of the precipitate growth formalism is based on the thermodynamic extremal principle of maximum entropy production [29]. The numerical time integration procedure for the evolution equations is similar to the classical numerical Kampmann - Wagner model [30]. Nucleation of precipitates is calculated from an extended Classical Nucleation Theory (CNT) [31,32] model for multicomponent systems. The transient nucleation rate $J$,

$$
J=N_{0} Z \beta^{*} \cdot P_{\text {nuсl }} \cdot e^{\left(-\frac{\tau}{t}\right)}
$$

describes the rate at which nuclei are formed per one unit volume and time. The unity is $\left[(\right.$ new nuclei $\left.) /\left(\mathrm{m}^{3} \mathrm{~s}\right)\right]$. In Equation (1), $N_{0}$ represents the total number of possible nucleation sites. The Zeldovich factor $Z$, takes into account that the nucleus is destabilized by thermal vibrations compared to the inactivated state and it is often of the order of $1 / 40$ to $1 / 100$. The atomic attachment rate $\beta^{*}$ takes into account the long-range diffusive transport of atoms, which is necessary for nucleus formation if the chemical composition of the matrix is different from the chemical composition of the precipitate. Furthermore these factors are multiplied by a factor $P_{\text {nucl }}$, which is related to the probability of a single nucleation event

$$
P_{\text {nucl }}=e^{\left(-\frac{G^{*}}{k \cdot T}\right)}
$$


and an expression which takes the incubation time $\tau$ into account.

$$
\tau=\frac{1}{2 \beta^{*} Z^{2}}
$$

In Equation (2), $k$ is the Boltzmann constant and $T$ is the absolute temperature. The critical nucleation energy $G^{*}$ is given by

$$
G^{*}=\frac{16 \pi}{3} \frac{\gamma^{3}}{D^{2}}
$$

which is a function of the interfacial energy $\gamma$ and the total driving force $D$.

Subsequently, a series of calculations for various cooling rates is presented and compared with the experiments. Finally, two parameter studies are shown with the aim of investigating the sensibility of the simulation results to the input data, i.e. to the accuracy of the independent thermodynamic and diffusion databases $[33,34]$, which we used for our calculations.

\section{Results}

\section{$\underline{\text { Precipitate morphology }}$}

The $\gamma^{\prime}$ precipitates observed in this study cover shapes from simple spheres over cubes, octocubes to very complex structures like octodendrites and dendrites. This transition is caused by the difference in lattice parameter of precipitate and matrix. This phenomenon is well known and it has been investigated several times (e.g. refs. [1,2,19]). Depending on the cooling rates between $78 \mathrm{~K} / \mathrm{s}$ and $0.0042 \mathrm{~K} / \mathrm{s}$, different morphologies of $\gamma$ ' precipitates in UDIMET 720 Li develop. Corresponding micrographs are shown in Figure 1.

Figure 1(a) shows precipitates, which form during cooling from $1180{ }^{\circ} \mathrm{C}$ to $400{ }^{\circ} \mathrm{C}$ with a rate of $78 \mathrm{~K} / \mathrm{s}$. The shape of these particles is spherical or at least nearly spherical. Lower cooling rates cause an increase in the mean particle diameter as shown in Figure 1(b). On further decreasing the cooling rate, the morphology of the microstructure gradually changes to more and more irregular precipitate shapes caused by the increasing misfit strain between particle and matrix with increasing precipitate size [2]. Figure 1(c) shows several non-spherical, facetted particles. In the lower right corner of the image, a precipitate with cubic cross section is observed. Another feature visible in this image is the observation of a second population of much smaller precipitates located in the corridors between the cubic particles. These multimodal particle distributions in UDIMET $720 \mathrm{Li}$ on continuous cooling have been reported previously by, e.g., Mao et al. [20] and they are also predicted by the present numerical simulations with MatCalc. If the cooling rate is further decreased, more and more cubes and octocubes are observed (Figures 1d,e). Finally, at the lowest cooling rates, the precipitate - matrix phase boundaries become incoherent and the precipitate shape resembles that of dendrites (Figure 1f).

The elongated precipitate shape at grain boundaries observed in sample 10 (Figure 1e) has stimulated further investigations. These shapes are produced by discontinuous precipitation, which occurs by the simultaneous precipitation at grain boundaries and grain boundary movement [35]. The right part of this image shows a very homogeneous distribution of precipitates with almost identical diameter, which is the result of a two-dimensional projection of a three-dimensional array of tubular precipitates in the normal to the growth direction of the precipitates. Figure 2 shows a corresponding microstructure reproduction performed with sequential sectioning using the FIB (focused ion beam) technique [36].

\section{Precipitate size evolution}

The samples 1-8 (spherical or nearly spherical precipitates), are also studied quantitatively with regard to the mean diameter. Since the determination of sizes and size distributions of precipitates with non-spherical morphologies is difficult, the quantitative analysis concentrates on samples with a mean precipitate diameter of less than $0.7 \mu \mathrm{m}$. This limit is well established in literature (see, e.g. ref. [37]). The measured and corrected mean diameters are summarized in Table III.

Table III: Measured and stereologically corrected [24]

\begin{tabular}{|c|c|c|c|}
\multicolumn{4}{|c|}{ precipitate sizes } \\
\hline \multirow{2}{*}{ sample } & \multirow{2}{*}{$\begin{array}{c}\text { cooling rate } \\
{[\mathrm{K} / \mathrm{s}]}\end{array}$} & \multicolumn{2}{|c|}{$\begin{array}{c}\mathrm{d}_{\text {mean }} \\
{[\mathrm{nm}]}\end{array}$} \\
\cline { 3 - 4 } & & measured & corrected \\
\hline 1 & 78 & 49.4 & 53.2 \\
2 & 19.5 & 58.6 & 63.8 \\
3 & 6.5 & 92.5 & 98.7 \\
4 & 3.25 & 149.6 & 159.8 \\
5 & 1.625 & 165.8 & 186.4 \\
6 & 0.867 & 258.4 & 278.9 \\
7 & 0.433 & 319 & 347.9 \\
8 & 0.217 & 379.9 & 393.5 \\
\hline
\end{tabular}

To complement the investigations, precipitation kinetics simulations are carried out with the software MatCalc. Depending on the applied cooling rate, the calculations predict the development of multi-modal particle distributions. The reason is found in the balance between continuously increasing supersaturation with decreasing temperature and the decreasing diffusivity of the precipitate-forming solute atoms. The interaction of these two counteracting mechanisms leads to the occurrence of 'nucleation waves'. 


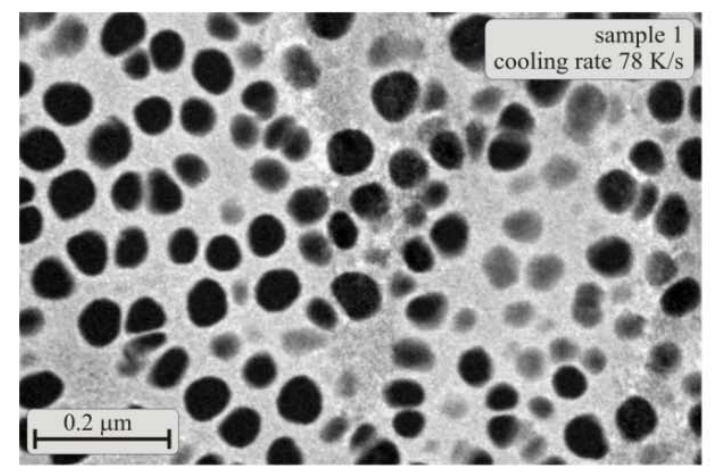

(a)

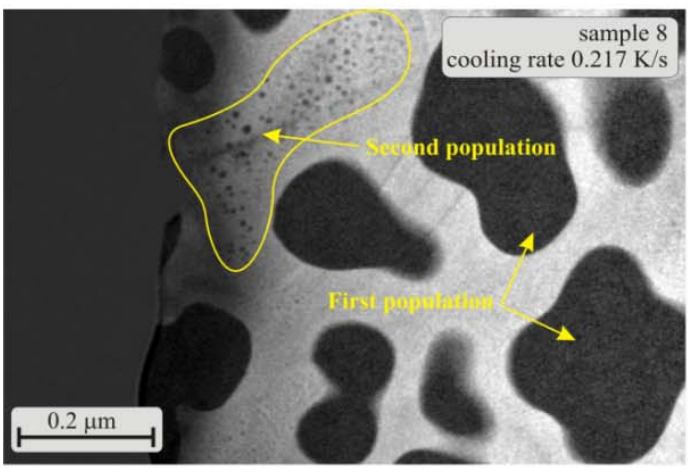

(c)

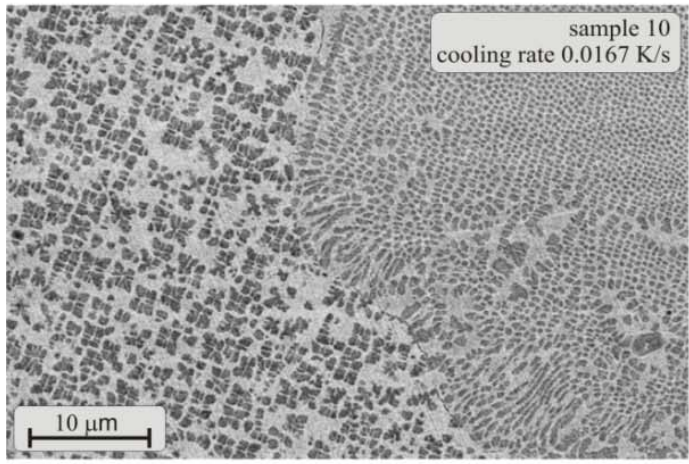

(e)

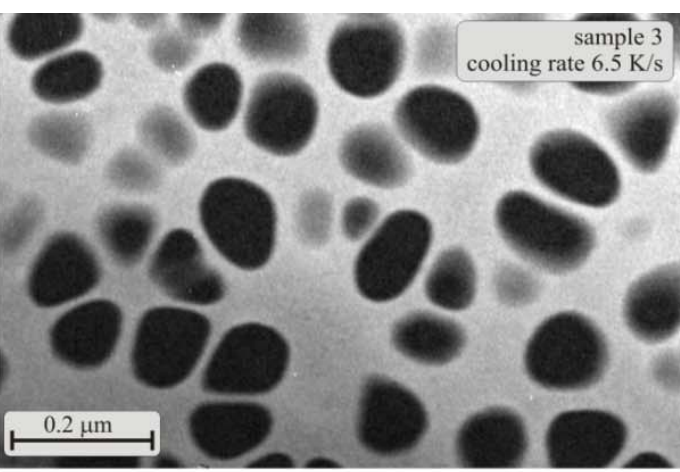

(b)

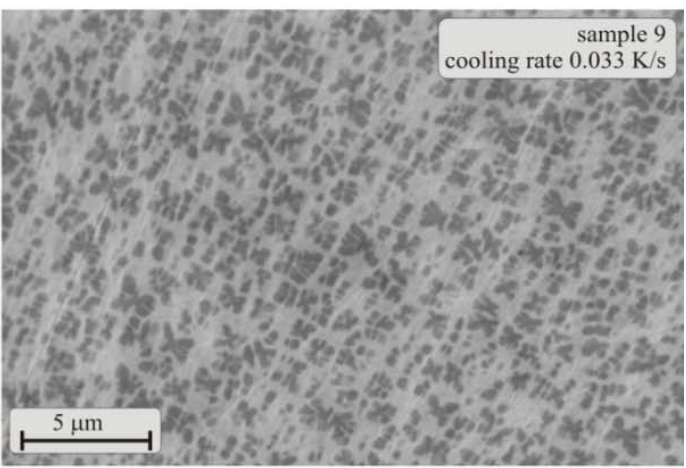

(d)

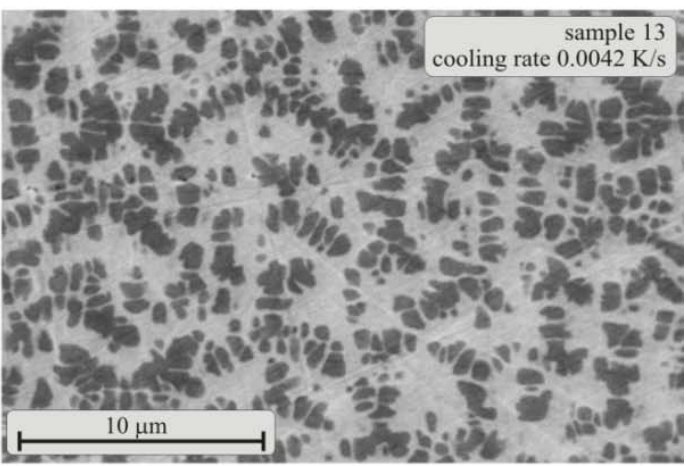

(f)

Figure 1: Morphologies of $\gamma^{\prime}$ precipitates in UDIMET $720 \mathrm{Li}$ depending on cooling rates between $78 \mathrm{~K} / \mathrm{s}$ and $0.0042 \mathrm{~K} / \mathrm{s}$ (a-c are EFTEM Cr-map images, $d-f$ are SEM images)

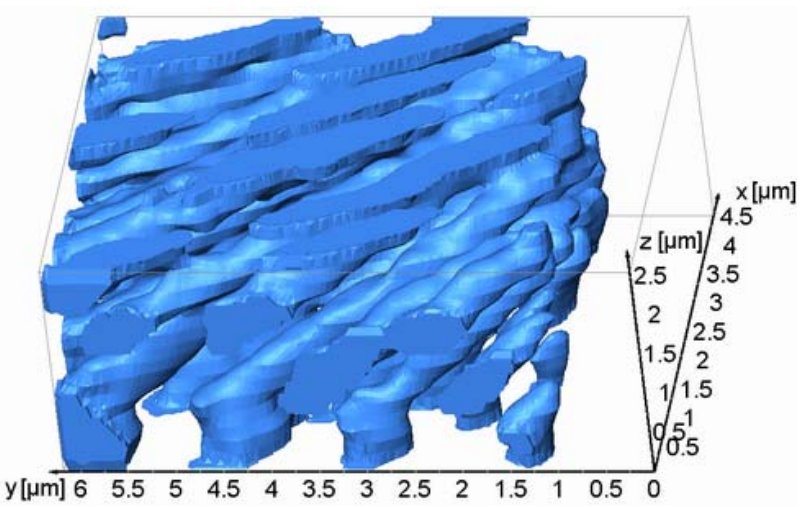

Figure 2: 3D-reconstruction of precipitates at the grain boundary using the method FIB 


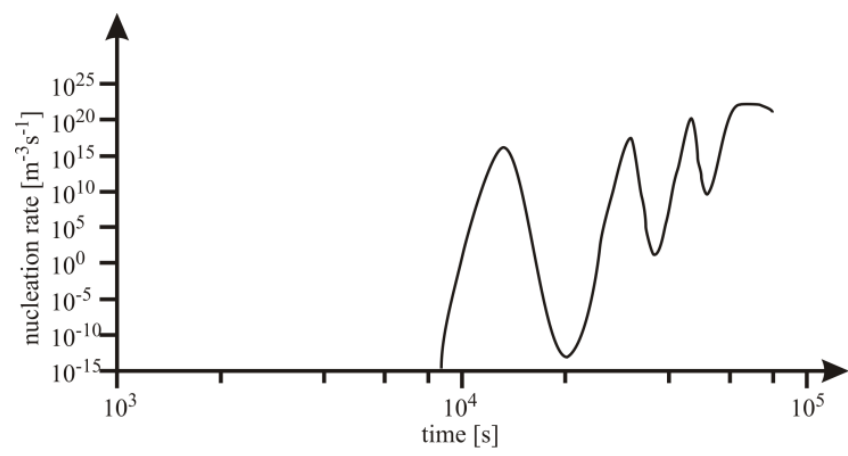

(a)

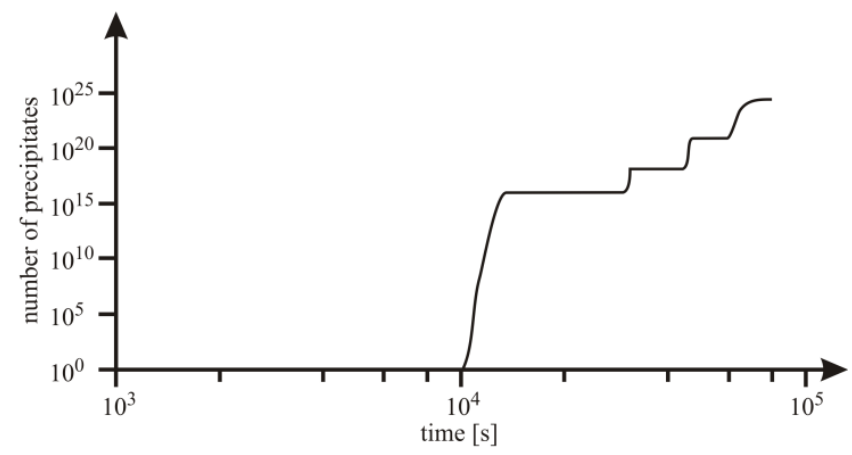

(b)

Figure 3: Calculated nucleation rate (a) and number of precipitates (b) while continuous cooling from $1180{ }^{\circ} \mathrm{C}$ to $400{ }^{\circ} \mathrm{C}$ in $79200 \mathrm{~s}$
Figure 3(a) shows a plot of the calculated nucleation rates demonstrating these oscillations. Figure 3(b) displays the predicted number of particles during cooling from $1180^{\circ} \mathrm{C}$ to 400 ${ }^{\circ} \mathrm{C}$. With each peak in the nucleation rate, the number of precipitates increases sharply. Due to these nucleation waves, various different precipitate size distributions are generated.

\section{Discussion}

Formation of multi-modal particle distributions during continuous cooling

Compared with isothermal heat treatments, the precipitation process during continuous cooling heat treatments is more complex. Generally, precipitation occurs in the three stages nucleation, growth and coarsening. In addition to the decrease of supersaturation in the matrix due to the formation of $\gamma^{\prime}$ precipitates, supersaturation of the matrix increases continuously due to the reduction of temperature. These two effects are counteracting mechanisms, which finally control the evolution of the multimodal precipitate distributions In addition to this, the diffusivity of elements also decreases with decreasing temperature, thus making elements less and less mobile and the maximum diffusion field extension smaller and smaller. Mathematically, all these processes enter the expressions for the critical nucleation barrier $G^{*}$ decreases (see Equation (4)) and the nucleation rate $J$ (Equation 1). The competition between increasing driving force, decreasing diffusivity and narrowing of the diffusion fields finally leads to the observed oscillating nucleation characteristics.

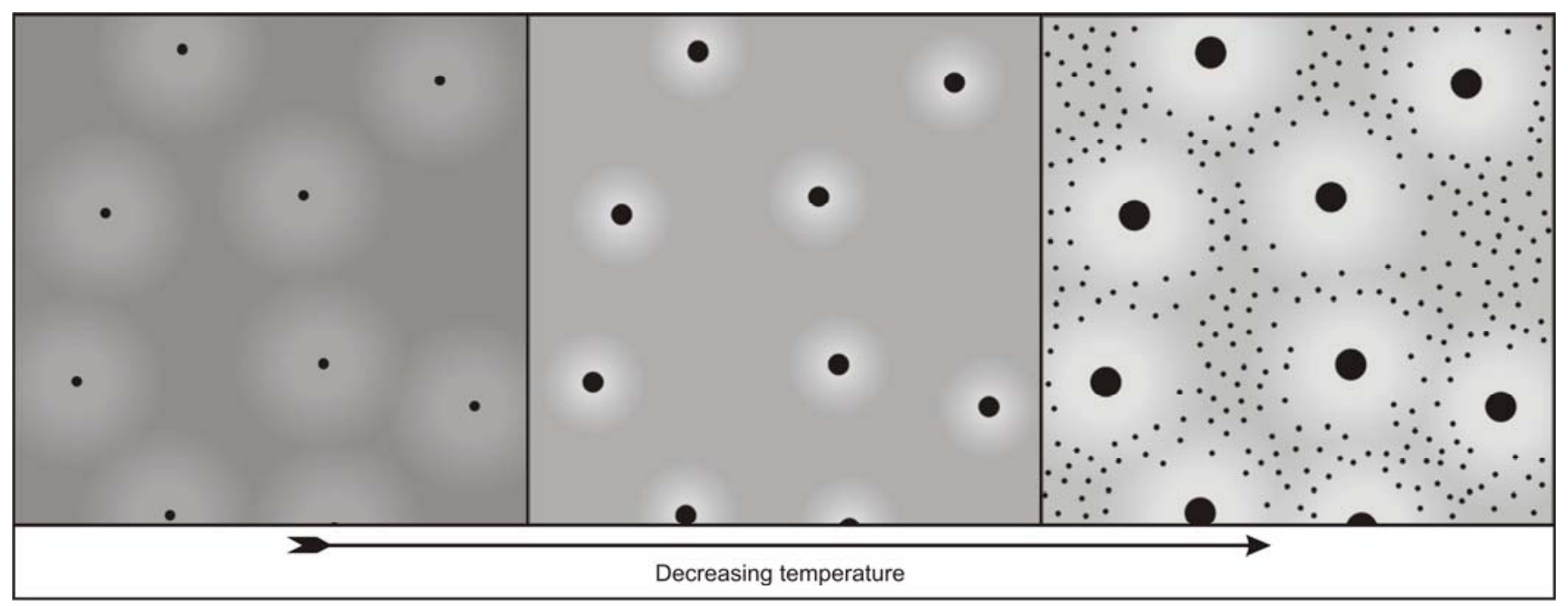

(a)

(b)

(c)

Figure 4: Competition between increasing supersaturation due to continuous cooling and decreasing supersaturation due to the formation of $\gamma^{\prime}$ precipitates

Figure 4 schematically shows the different stages of the evolution of multi-modal distributions during continuous cooling. After cooling below solid solution temperature, a first nucleation burst of $\gamma^{\prime}$ precipitates occurs, characterized by low supersaturation and thus low nucleation rate. Figure 4(a) indicates that the particle spacing in the first nucleation wave is wide. At elevated temperature, the $\gamma^{\prime}$ precipitates are surrounded by large diffusion fields, causing extensive growth to large size.
On decreasing temperature, the maximum diffusion field extension reduces and, in the corridors between the existing precipitates of the first nucleation burst, the supersaturation in the matrix increases again (Figure 4(b)). This causes another nucleation burst at lower temperatures and a second $\gamma^{\prime}$ distribution of precipitates in between the precipitates of the first wave (Figure 4(c)). Because the diffusivity is much smaller at lower temperatures, the precipitates of the second wave grow slower and the mean precipitate size also remains much smaller. By further 
continuous cooling another critical temperature can be achieved which eventually leads to a third nucleation burst and hence to a third population of $\gamma^{\prime}$ precipitates between the precipitates of the first and the second population. This process repeats until no further nucleation occurs. The simulation as well as the experimental observation clearly demonstrate this effect.

Comparison between theoretical predictions and experimental $\underline{\text { results }}$

Figure 5 summarizes and compares the experimental and computational results where the three solid lines represent the three predicted precipitate populations. The dashed lines indicate regions where overlapping of distributions is predicted. From these results it is easily seen that the mean diameter of precipitates increase linearly with cooling time if plotted in double logarithmic scale. This confirms the observations of Mao et.al. [20], who derived a phenomenological relation (Equation 7) for the size of precipitates of the first population as a function of cooling rate.

$$
\log D_{\gamma^{\prime}}=0.0165-0.354 \cdot \log \frac{d T}{d t}
$$

Very good agreement between the experimental data of the present work and of the data of Mao et.al. [20] is observed.

It is important to emphasize that the present simulations are predictive in character and no fitting parameters are employed. In this sense, prediction and experiment can be considered to be in satisfying agreement over a wide range of different cooling rates, at least for the first population.

Despite the correct prediction of the size of the primary precipitate population, deviations in the second and third population are observed. The measured sizes for the second and third population are generally too small compared to the simulations. The differences between simulation and experimental observation can be attributed to a number of simplifying assumptions in the model as well as difficulties in the measurement procedures:

- The precipitation kinetics model implemented in MatCalc is a mean-field type model and it is therefore strictly valid only for dilute solution, where the size of the precipitates are assumed to be sufficiently small compared to the diffusion fields surrounding the precipitates. This is certainly not valid in the present UDIMET $720 \mathrm{Li}$ alloy due to the high amount of alloying elements. Often, the precipitates are located very close to each other and the corridors between the precipitates for other populations are too narrow.

- Both, the simulation with "MatCalc" and also the correction method after Sonderegger [27] require spherical shape of precipitates. This prerequisite is not entirely fulfilled for larger precipitates.

Furthermore, it should be noted that theoretical predictions can only be as exact as the accuracy of the input data, i.e. the thermodynamic and diffusion databases [33,34]. To investigate the sensitivity of the simulation results on variations of input data, two parameter studies are carried out for the most sensitive and, probably, also most undetermined parameters interfacial energy and diffusivity of alloying elements. Both parameters are varied by constant factors.

Figure 6(a) shows a distinct maximum of the predicted mean precipitate size when varying the value of the predicted interfacial energy. The maximum is observed when multiplying the predicted interfacial energy by a factor of 0.8. A linear dependence of the mean precipitate size is observed on the diffusion coefficient (Figure 6(b)), if the curves are plotted in double logarithmic scale. This means that the precipitate size scales directly with the diffusion coefficient. A change in the diffusion coefficient by a factor of two causes a variation in the mean precipitate size by a factor of 1.5 .

This parameter studies show in a realistic manner the strong dependence of the simulation on the accuracy of the input data. Furthermore, it should be noted that these parameters are experimentally determined and uncertainties in the diffusion coefficient by a factor of two are realistic. Concerning these facts, the accuracy in predicting the precipitate sizes must be considered as excellent. It is again emphasized that no fitting parameters have been employed in the simulations and all values have been taken from the independent thermodynamic and diffusion databases $[33,34]$.

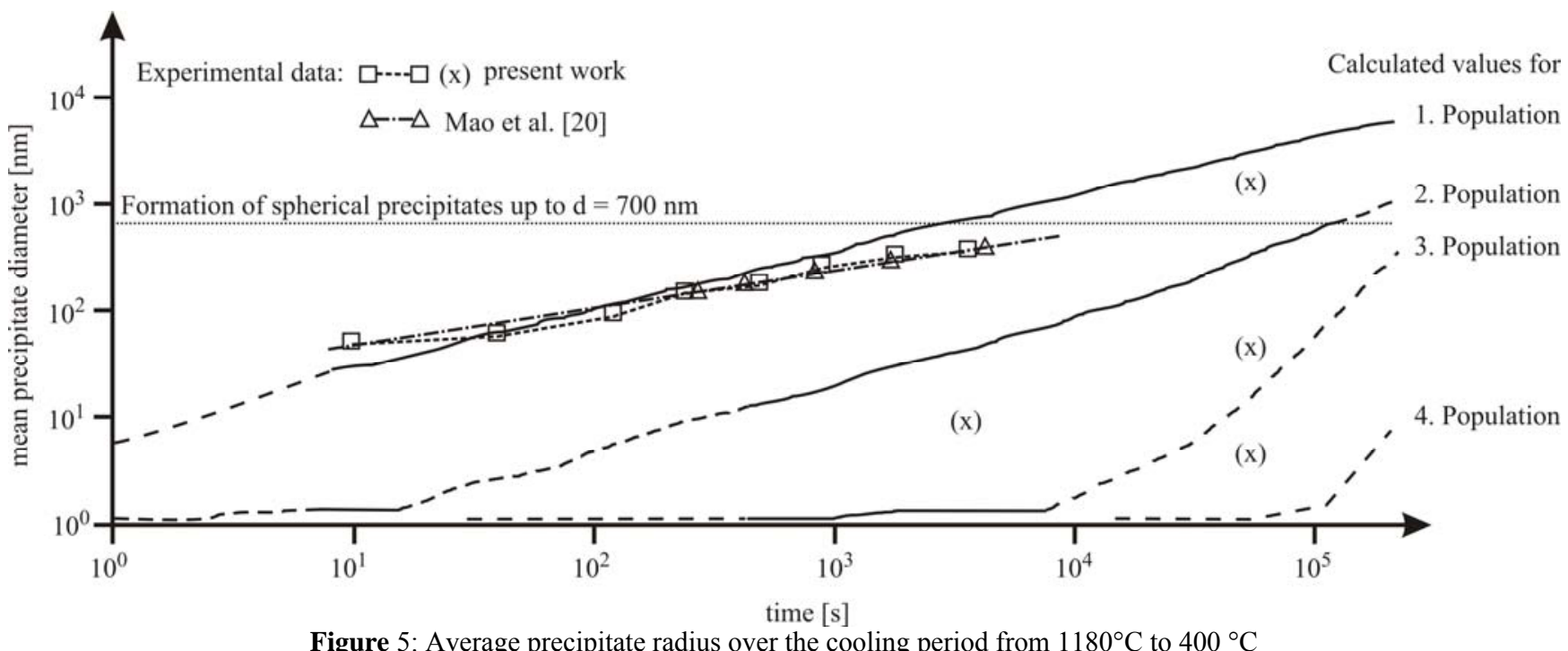

Figure 5: Average precipitate radius over the cooling period from $1180^{\circ} \mathrm{C}$ to $400{ }^{\circ} \mathrm{C}$ 


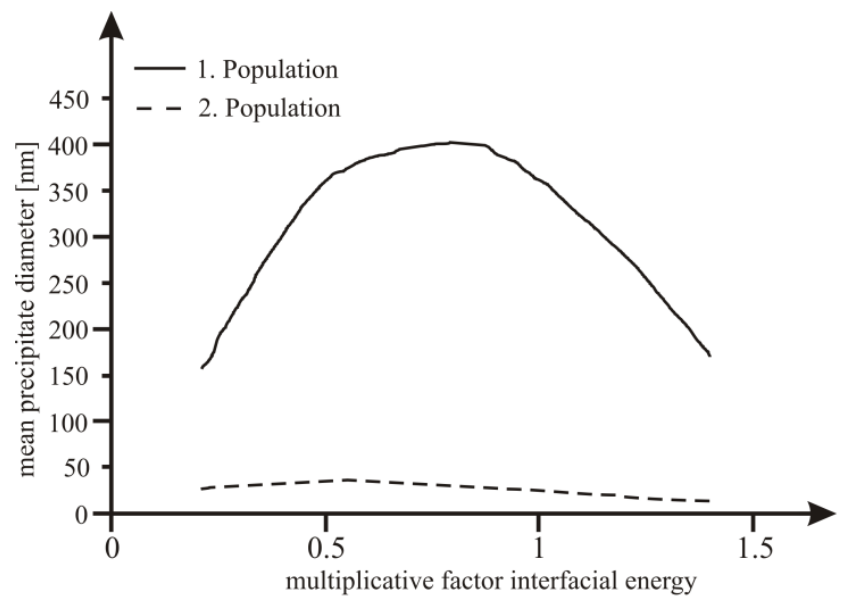

(a)

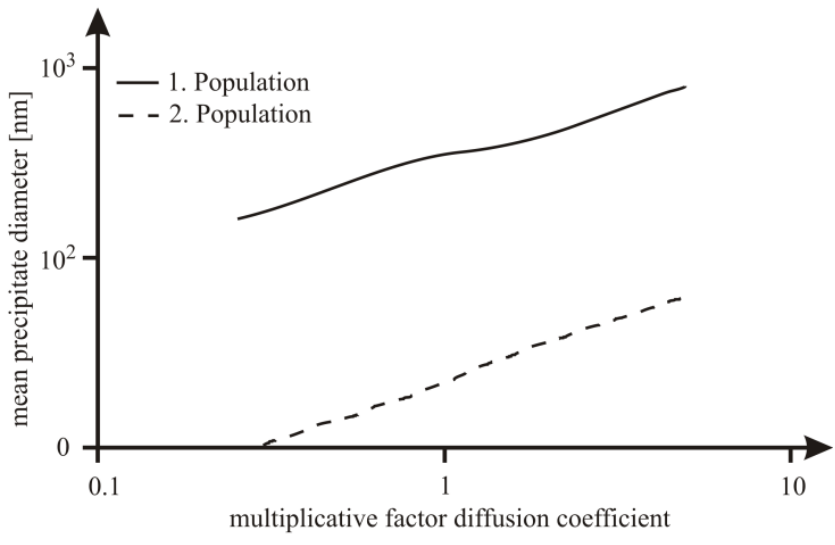

(b)

Figure 6: Parameter studies concerning the interfacial energy (a) and the diffusion coefficient (b)

\section{Summary and Conclusions}

- On continuously cooling samples from the alloy UDIMET $720 \mathrm{Li}$, depending on cooling rate, discontinuous precipitation as well as mono-modal and multi-modal distributions are observed.

- With decreasing cooling rate, the morphology of $\gamma^{\prime}$ precipitates in UDIMET $720 \mathrm{Li}$ change from spheres over cubes and octocubes to incoherent dendritic structures (see also ref. [2] for other nickel based superalloys).

- The theoretically predicted average precipitate size and the experimental diameter of the $\gamma^{\prime}$ precipitates of the present work and of the work [20] are in excellent agreement over a wide range of cooling rates, at least for the first population.

- On a logarithmic scale, the average precipitate radius versus cooling time shows an approximately linear relation. This is in agreement with the observations in ref. [20].

- The theoretically predicted multi-modal precipitate distributions are experimentally confirmed.

- The parameter studies on the influence of the thermodynamic and diffusion data on the simulation results clearly indicate a strong dependence of the results from the input data. Particularly important seems to be accurate data on the diffusion coefficients of the alloying elements over the entire range of temperatures.

\section{Acknowledgement}

Financial support by the Österreichische Forschungsförderungsgesellschaft $\mathrm{mbH}$, the Province of Styria, the Steirische Wirtschaftsförderungsgesellschaft $\mathrm{mbH}$ and the Municipality of Leoben within research activities of the Materials Center Leoben under the frame of the Austrian Kplus Competence Center Programme is gratefully acknowledged.

\section{References}

[1] R.A. Ricks, A.J. Porter and R.C. Ecob, "The Growth of $\gamma$ ' Precipitates in Nickel-Base Superalloys", Acta Metall., 31 (1983), 43-53.

[2] T. Grosdidier, A. Hazotte and A. Simon, "Precipitation and Dissolution Processes in $\gamma / \gamma$ ' Single Crystal Nickel-Based Superalloys", Mat. Sci. Eng., A256 (1998), 183-196.

[3] E. Hornbogen and M. Roth, "Die Verteilung kohärenter Teilchen in Nickellegierungen“" Z. Metallkd., 58 (1967), 842-855.

[4] T. Maebashi and M. Doi, "Coarsening Behaviours of Coherent $\gamma$ ' and $\gamma$ Precipitates in Elastically Constrained Ni-Al-Ti Alloys", Mat. Sci. Eng., A373 (2004), 72-79.

[5] A. Maheshwari and A.J. Ardell, "Anomalous Coarsening Behavior of Small Volume Fractions of $\mathrm{Ni}_{3} \mathrm{Al}$ Precipitates in Binary Ni-Al Alloys, Acta Metall. Mater"., 40(10) (1992), 26612667.

[6] T. Miyazaki, K. Nakamura and H. Mori, "Experimental and Theoretical Investigations on Morphological-Changes of $\gamma^{\prime}$ Precipitates in Ni-Al Single-Crystal During Uniaxial StressAnnealing”, J. Mater. Sci., 14(8) (1979), 1827-1837.

[7] T. Miyazaki, H. Imamura, H. Mori and T. Kozakai, "Theoretical and Experimental Investigations on Elastic Interactions Between $\gamma$ '-Precipitates in A Ni-Al Alloy", J. Mater. Sci., 16(5) (1981), 1197-1203.

[8] A. Hazotte, T. Grosdidier and S. Denis, " $\gamma$ ' Precipitate Splitting in Nickel-Based Superalloys: A 3-D Finite Element Analysis", Scripta Mater., 34(4) (1996), 601-608.

[9] M. Doi and T. Miyazaki, “ $\gamma$ '-Precipitate Morphology Formed Under the Influence of Elastic Interaction Energies in NickelBased Alloys", Mat. Sci. Eng., 78(1) (1986), 87-94.

[10] H. Monajati, M. Jahazi, R. Bahrami and S. Yue, „The Influence of Heat Treatment Conditions on $\gamma$ ' Characteristics in UDIMET 720”, Mat. Sci. Eng., A 373 (2004), 286-293.

[11] T. Grosdidier, A. Hazotte and A. Simon; "On the Dissolution Mechanisms of $\gamma$ '-precipitates in Nickel-Based Superalloys", Scripta Metall. Mater., 30(10) (1994), 1257-1262.

[12] M.P. Jackson and R.C. Reed, "Heat Treatment of UDIMET 720Li: The Effect of Microstructure on Properties", Mat. Sci. Eng., A259 (1999), 85-97.

[13] F. Torster, G. Baumeister, J. Albrecht, G. Lütjering, D. Helm and M.A. Daeubler, "Influence of Grain Size and Heat Treatment 
on the Microstructure and Mechanical Properties of the NickelBase Superalloy U 720 Li, Mat. Sci. Eng., A234-236 (1997), 189192.

[14] H.J. Penkalla, J. Wosik and A. Czyrska-Filemonowicz, "Quantitative Microstructural Characterization of Ni-Base Superalloys, Mater. Chem. Phys., 81 (2003), 417-423.

[15] P.M. Sarosi, B. Wang, J.P. Simmons, Y. Wang and M.J. Mills, "Formation of Multimodal Size Distributions of $\gamma$ ' in a Nickel-Base Superalloy During Interrupted Continuous Cooling”, Scripta Mater., 57 (2007), 767-770.

[16] S.S. Babu, M.K. Miller, J.M. Vitek and S.A. David, "Characterization of the Microstructure Evolution in a NickelBase Superalloy During Continuous Cooling Conditions", Acta. Mater., 49 (2001), 4149-4160.

[17] J.P. Simmons, Y. Wen, C. Shen and Y.Z. Wang, "Microstructural Development Involving Nucleation and Growth Phenomena Simulated with the Phase Field Method", Mat. Sci. Eng., A365 (2004), 136-143.

[18] Y.H. Wen, J.P. Simmons, C. Shen, C. Woodward, Y.Wang, "Phase-Field Modeling of Bimodal Particle Size Distributions During Continuous Cooling”, Acta Mater., 51 (2003), 1123-1132.

[19] D.U. Furrer and H.J. Fecht, " $\gamma$ ' Formation in Superalloy U720Li“, Scripta Mat., 40(11) (1999), 1215-1220.

[20] J. Mao, K.M. Chang, W. Yang, K. Ray, S.P. Vaze and D.U. Furrer, "Cooling Precipitation and Strengthening Study in Powder Metallurgy Superalloy U720Li”, Metall. Trans. A., 32(10) (2001), 2441-2452.

[21] J. Mao, K.M. Chang, W. Yang, D.U. Furrer, K. Ray and S.P. Vaze, "Cooling Precipitation and Strengthening Study in Powder Metallurgy Superalloy Rene88DT”, Mat. Sci: Eng., A332 (2002), 318-329.

[22] T.P. Gabb, D.G. Backman, D.Y. Wie, D.P.Mourer, D. Furrer, Anita Garg and D.L. Ellis, “ $\gamma$ ' Formation in a Nickel-Base Disk Superalloy”, Proc. in Superalloys 2000, TMS (2000), 405-414.

[23] J. Svoboda, F.D. Fischer, P. Fratzl and E. Kozeschnik, "Modelling of Kinetics in Multi-Component Multi-Phase Systems with Spherical Precipitates I. - Theory", Mater. Sci. Eng., A385 (2004), 166-174.

[24] E. Kozeschnik, J. Svoboda, P. Fratzl and F.D. Fischer, "Modelling of Kinetics in Multi-Component Multi-Phase Systems with Spherical Precipitates II. - Numerical Solution and Application”, Mater. Sci. Eng., A385 (2004), 157-165.

[25] E. Kozeschnik, J. Svoboda and F.D. Fischer, "Modified Evolution Equations for the Precipitation Kinetics of Complex Phases in Multi-Component Systems", CALPHAD, 28 (4) (2005), 379-382.

[26] M. Stockinger, "Mikrostrukturelle Simulation des Gesenkschmiedens von Nickelbasis - Legierungen" (Ph.D. thesis, Technische Universität Graz - Austria, 2003).
[27] B. Sonderegger, "Modifications of Stereological Correction Methods for Precipitation Parameters using Transmission Microscopy“, Ultramicroscopy, 107 (2007), 587-597.

[28] P.F.Schmidt, "Praxis der Rasterelektronenmikroskopie und Mikrobereichsanalyse“ (Münster - Germany: Expert Verlag, 1994).

[29] Svoboda J; Turek I; Fischer FD; "Application of the Thermodynamic Extremal Principle to Modeling of Thermodynamic Processes in Material Sciences", Philosophical Magazine, 85(31) (2005), 3699-3707.

[30] R. Kampmann and R. Wagner, "Kinetics of Precipitation in Metastable Binary Alloys - Theory and Applications", Acta Scripta Metall. series, "Decomposition of Alloys: the Early Stages" (1984), 91-103.

[31] K.G.F. Janssens, D. Raabe, E. Kozeschnik, M.A. Miodownik and Britta Nestler, "Computational Materials Engineering - An Introduction to Microstructure Evolution" (Oxford - UK: Elsevier Academic Press 2007).

[32] K. Russell, "Nucleation in Solids: The Induction and Steady States", Adv. Colloid. Sci., 13 (1980), 205-318.

[33] ThermoTech, Ni-Base Version 3.0 http://www.thermotech.co.uk/databases.html (01.08.07).

[34] C.E. Campbell, W.J. Boettinger, and U.R. Kattner, "Nickel Mobility Database by C.E. Campbell”, Acta Mater., 50 (2002), 775-792.

[35] D.A. Porter and K.E. Easterling, "Phase Transformations in Metals and Alloys" (United States of America - Boca Raton: CRC Press, 2004).

[36] M. Schaffer, J. Wagner, B. Schaffer, M. Schmied and H. Mulders, "Automated Three-Dimensional X-ray Analysis Using a Dual-Beam FIB”, Ultramicroscopy, 107 (2007), 587-597.

[37] E. Nembach and G. Neite, "Precipitation Hardening of Superalloys by Orderd $\gamma$ '-Particles”, Mat. Sci., 29 (1985), 177319. 